derived publication will explicitly mention the origin of the samples used; and 2) to return to the CPBC the raw data of their experiments, once the publication is online. This is an important and innovative condition that we hope will transform the CPBC from a passive collection of samples and information into an active and growing source of knowledge, which will be then made freely available to future users. The $\mathrm{CPBC}$, therefore, offers a unique opportunity to enhance translational research of excellence in respiratory diseases within Europe and beyond.

\section{Villena*, F. Pozo", J.A. Barberà , P. Vaquer* and}

\section{A. Agusti ${ }^{\top}$ on behalf of the CIBERES Pulmonary Biobank} Consortium (PBC) ${ }^{+}$

*Centro de Investigación Biomédica en Red de Enfermedades Respiratorias (CIBERES), Mallorca, "Hospital Universitario Doce de Octubre, Madrid, and "Servei de Pneumologia, Institut del Tòrax, Hospital Clinic-IDIBAPS, Barcelona, Spain. ${ }^{+}$The CIBERES Pulmonary Biobank Consortium (CPBC) participants are: M. Arque and C. Villena (coordinator; both Centro de Investigación Biomédica en Red de Enfermedades Respiratorias (CIBERES), Mallorca, Spain); J. Cortijo, E. Donet, R. Guijarro, G. Juan, J. Lluch and M. Martorell (all Consorcio Hospital General Universitario de Valencia, Valencia, Spain); G. Peces-Barba, G. Renedo, M.J. Rodriguez-Nieto and J. Zapatero (all Fundación Jiménez-Díaz-Capio, Madrid, Spain); J.A. Barberà, L. Molins, V. Peinado and J. Ramírez (all Hospital Clinic, Barcelona, Spain); E. Condom, S. Estany, M. Molina, J. Moya and A. Rosell (all Hospital Universitari de Bellvitge, Barcelona); E. Camarero, M. Casares, B. de Olaiz, A. Esteban, L. Fernandez- Jiménez and P. Segoviano (all Hospital Universitario de Getafe, Madrid); C. Casadevall, J. Gea, M.A. Martínez, J. Minguella and L. Pijuan (Hospital Universitario del Mar, Barcelona); E.M. Arias, A.B. Enguita, A. López-Encuentra, A. Maroto, C. Marrón, F. Pozo and J.L. Rodriguez (all Hospital Universitario Doce de Octubre, Madrid); J. Astudillo, M.T. Fernández-Figueras, E. Monsó and S. Ragull (all Hospital
Universitario Germans Trias i Pujol, Badalona, Spain); E. Canalis, I. Martín-Loeche, J. Rello, J.J. Sirvent and S. Trefler (all Hospital Universitario Joan XXIII de Tarragona, Tarragona, Spain); M. Arque, A. Carvajal, B. Cosio, C. Gómez, J. Salueda and C. Villena (Hospital Universitario Son Dureta, Mallorca).

Correspondence: A. Agusti, Institut del Tòrax, Hospital Clínic, C/Villarroel 170, Escala 3, Planta 5, 08036 Barcelona, Spain. Email: alvar.agusti@clinic.ub.es

Statement of Interest: None declared.

\section{REFERENCES}

1 Riegman $\mathrm{PH}$, Morente MM, Betsou F, et al. Biobanking for better healthcare. Mol Oncol 2008; 2: 213-222.

2 Founti P, Topouzis F, van Koolwijk L, et al. Biobanks and the importance of detailed phenotyping: a case study: the European Glaucoma Society GlaucoGENE project. Br J Ophthalmol. 2009; 93: 577-581.

3 Agusti A, Pozo F, Vaquer P. [CIBER of respiratory diseases (CibeRes)]. Arch Bronconeumol 2008; 44: 489-492.

4 ISBER, Best Practices for Repositories: Collection, Storage, Retrieval and Distribution of Biological Materials for Research. Cell Preserv Technol 2008; 6: 3-58.

5 Organisation for Economic Co-operation and Development. OECD Best Practice Guidelines for Biological Resources Centres. www. oecd.org/dataoecd/7/13/38777417.pdf Date last accessed: October 18, 2010. Date last updated: 2007.

6 LEY 14/2007, de 3 de julio, de Investigación biomédica [Biomedical Research Act 14/2007, July 3]. Boletin Oficial del Estado, 159: 28826-28848.

7 Mager SR, Oomen MH, Morente MM, et al. Standard operating procedure for the collection of fresh frozen tissue samples. Eur J Cancer 2007; 43: 828-834.

8 National Cancer Institute. Best Practices for Biospecimen Resources. http://biospecimens.cancer.gov/global/pdfs/

NCI_Best_Practices_060507.pdf Date last accessed: October 18, 2010 Date last update: June 2007.

\title{
Dose-response for inhaled fluticasone on airway and systemic inflammation in COPD
}

\section{To the Editors:}

Asthma is an eosinophilic condition and, as such, responds readily to inhaled corticosteroids (ICS). By contrast, chronic obstructive pulmonary disease (COPD) is a heterogeneous condition associated with neutrophilic inflammation, which is relatively steroid-insensitive. Corticosteroid/long-acting $\beta$ agonist combination inhalers improve quality of life and lung function, and reduce COPD exacerbation [1]. A meta-analysis of COPD studies has reported a $25 \%$ reduction in all-cause mortality using ICS, but dose varied between 400 and
$1,000 \mu \mathrm{g} \cdot$ day $^{-1}$ of fluticasone propionate (FP) equivalent and no dose-response relationship was apparent [2]. There is, however, a dose-related risk of side-effects, including dysphonia, osteoporosis, cataracts and pneumonia [3,4]. Evidence is scant for an optimal ICS dose that confers long-term benefits whilst minimising potential risks.

COPD is now considered a systemic inflammatory disease. Biomarkers, such as C-reactive protein (CRP), are elevated in COPD and are associated with cardiovascular mortality and recurrent exacerbations [5]. It has been proposed that ICS may 
play a role in treating systemic inflammation. Proposed biomarkers of inflammation in COPD include: CRP, interleukin (IL)-6, tumour necrosis factor (TNF)- $\alpha$, surfactant protein (SP)D and exhaled markers, such as nitric oxide (NO). Measurement of the exhaled NO fraction (FeNO) at multiple flow rates can be used to derive values of $\mathrm{NO}$ production from conducting (bronchial flux; $J^{\prime}$ aw,NO) and small airways (alveolar nitric oxide; $\mathrm{CA}, \mathrm{NO}$ ). $\mathrm{CA}, \mathrm{NO}$ is elevated in COPD and is not effected by smoking [6]. Although studies have assessed biomarker response to fixed doses of ICS, dose response has not been addressed.

To explore the risk/benefit of ICS in COPD, we have performed a pilot study of the corticosteroid dose-response relationship on airway and systemic inflammatory outcomes.

Current or ex-smokers, $>50$ yrs of age, with a forced expiratory volume in $1 \mathrm{~s}$ (FEV1)/forced vital capacity ratio $<0.7$, FEV $1<80 \%$, and reversibility $<15 \%$ and $200 \mathrm{~mL}$ were enrolled in a double-blind crossover study. The exclusion criteria were asthma and bronchiectasis. Participants were randomised and grouped into treatment blocks of a 4-week dose ramp using $250 \mu \mathrm{g}$ FP (Cipla, Mumbai, India) or placebo. Dose ramps consisted of $500 \mu \mathrm{g} \cdot$ day $^{-1} \mathrm{FP}$, followed by $2,000 \mu \mathrm{g} \cdot \mathrm{day}^{-1}$ for 2 weeks each, or a corresponding placebo. There was a steroid-free run-in and a wash-out between treatments, each of 2 weeks. Measurements were performed at baseline, after run-in and washout, and at 2 and 4 weeks of each treatment. The study was sponsored by the University of Dundee (Dundee, UK), approved by the Tayside Committee on Medical Research Ethics (Dundee, UK) and registered at www.clinicaltrials.gov (NCT00995475).

Spirometry was performed in accordance with European Respiratory Society (ERS) guidelines. FeNO was measured at 50, 100 and $200 \mathrm{~mL} \cdot \mathrm{s}^{-1}$ (Niox; Aerocrine AB, Solna, Sweden). $\mathrm{CA}, \mathrm{NO}$ and $J^{\prime}$ aw,NO were derived using the two-compartment model [7]. Urine was collected overnight for $10 \mathrm{~h}$. Urinary creatinine was determined using a Roche Cobas analyser (Roche, Burgess Hill, UK; coefficient of variance (CV) 5.3\%) and cortisol by radioimmunoassay (DiaSorin, Bracknell, UK; CV 10.8\%). Biomarkers were determined using commercial ELISA kits for CRP (Kalon Biological Ltd, Guildford, UK; CV $16.2 \%$ ), IL-6 (Diaclone, Manchester, UK; CV 4.4\%), TNF- $\alpha$ (R\&D Systems Europe, Abingdon, UK; CV 6.5\%) and SPD (BioVendor, Heidelberg, Germany; CV 4.1\%).

Data were assessed for normality and nongaussian data were transformed prior to analysis. Values after run-in and washout periods were compared with a paired t-test to exclude carryover effects. Treatments were compared using ANOVA of repeated measures with planned contrasts between active treatments and for each active treatment compared to respective placebo. A priori calculations predicted an $80 \%$ power with 12 completed patients to detect a $50 \%$ reduction in CRP, with a two-tailed p-value $<0.05$, based on a study showing CRP suppression by ICS in COPD [8].

18 participants completed the study per protocol; five subjects were current smokers. Mean \pm SEM age and smoking exposure were $66 \pm 1.3$ yrs and $50 \pm 5.4$ pack-yrs, respectively. Mean \pm SEM FEV1 was $57 \pm 3.2 \%$ predicted. There was no suppression of CRP, IL-6 or TNF- $\alpha$ with 500 or $2,000 \mu \mathrm{g} \cdot$ day $^{-1}$ FP compared to placebo (table 1). There was a trend for suppression of SPD with fluticasone compared to placebo: 75 versus $91 \mathrm{ng} \cdot \mathrm{mL}^{-1}$ SPD for $500 \mu \mathrm{g} \cdot$ day $^{-1} \mathrm{FP}$ and 73 versus $85 \mathrm{ng} \cdot \mathrm{mL}^{-1} \mathrm{SPD}$ for 2,000 $\mu$ g.day ${ }^{-1}$ $\mathrm{FP}$, but neither was significant $(\mathrm{p}=0.13$ and $\mathrm{p}=0.28$, respectively). There was no difference in CRP, IL- 6 , TNF- $\alpha$ or SPD between active treatments. Early morning serum cortisol was suppressed by 500 and $2,000 \mu \mathrm{g} \cdot$ day $^{-1}$ FP compared to placebo. $10-\mathrm{h}$ overnight urinary cortisol excretion corrected for creatinine (OUCC) was suppressed with $2,000 \mu \mathrm{g} \cdot \mathrm{day}^{-1} \mathrm{FP}(\mathrm{p}<0.001)$ and a trend to suppression with $500 \mu$ g.day ${ }^{-1}$ FP $(p=0.08)$. There was a difference between 500 and 2,000 $\mu$ g.day ${ }^{-1} \mathrm{FP}$ for both serum cortisol $(\mathrm{p}=0.004)$ and OUCC $(\mathrm{p}=0.014)$. Compared to placebo, both 500 and 2,000 $\mu$ g.day ${ }^{-1}$ FP suppressed FeNO, J'aw,NO and CA,NO (table 1). There was no difference between doses.

This study is an initial step to investigate the dose response of inflammation in COPD. Multicentre trials assessing ICS have examined various steroid preparations and combination therapies, but none has examined the dose-response relationship of beneficial effects. Although it is considered that doses $\leqslant 500 \mu \mathrm{g} \cdot$ day $^{-1} \mathrm{FP}$ are relatively safe in asthma and COPD [9], each increase of $500 \mu \mathrm{g}$.day ${ }^{-1} \mathrm{FP}$ is associated with a $25 \%$ increased risk for cataracts, a $12 \%$ increased risk for nonvertebral fractures and a 1.4-fold risk for adrenal suppression [3, 4].

The current study employed a starting dose of $500 \mu \mathrm{g} \cdot$ day $^{-1} \mathrm{FP}$ as a "safe dose" for long-term therapy and compared this to a higher than usual dose of inhaled therapy; 2,000 $\mu \mathrm{g} \cdot$ day $^{-1} \mathrm{FP}$. Inhaled FP is commonly prescribed in COPD and was shown to suppress CRP in a pilot study by Sin et al. [8]. They demonstrated a $50 \%$ reduction following 2 weeks of $1,000 \mu \mathrm{g} \cdot \mathrm{day}^{-1}$ and a $63 \%$ reduction following $30 \mathrm{mg} \cdot \mathrm{day}^{-1}$ prednisolone, with parallel changes in IL-6. Our power calculations were based on this study; however, we did not demonstrate suppression of CRP, IL-6 or TNF- $\alpha$. SiN et al. [10] have subsequently performed a larger multicentre study comparing FP, FP/salmeterol and placebo. In contrast to their pilot, they showed no suppression of CRP or IL-6 with $1,000 \mu \mathrm{g} \cdot$ day $^{-1}$ FP, though they did not investigate whether suppression could be achieved at higher doses. The present study provides evidence that CRP and IL-6 are insensitive to ICS at commonly prescribed doses but, additionally, demonstrates there is not a clinically useful dose threshold above this at which suppression of systemic inflammation occurs, given that $2,000 \mu \mathrm{g} \cdot \mathrm{day}^{-1}$ is higher than commonly prescribed and was associated with a significant degree of adrenal suppression.

SIN et al. [10] demonstrated a reduction in SPD, which is a lungspecific biomarker produced in response to pneumocyte injury. Elevated systemic levels are associated with atherosclerosis. Although we did not demonstrate a statistically significant suppression, the numerical trend was similar to the results of SIN et al. [10]. Modest drug response and wide interindividual variation suggest that, even if SPD has a pathological role in atherosclerosis in COPD, it will not be sensitive enough to guide routine clinical practice on an individual basis.

In addition to systemic inflammatory markers, the current study included airway inflammatory markers in the form of extended exhaled nitric oxide. We hypothesised that, if systemic inflammation were suppressed, this might offer 


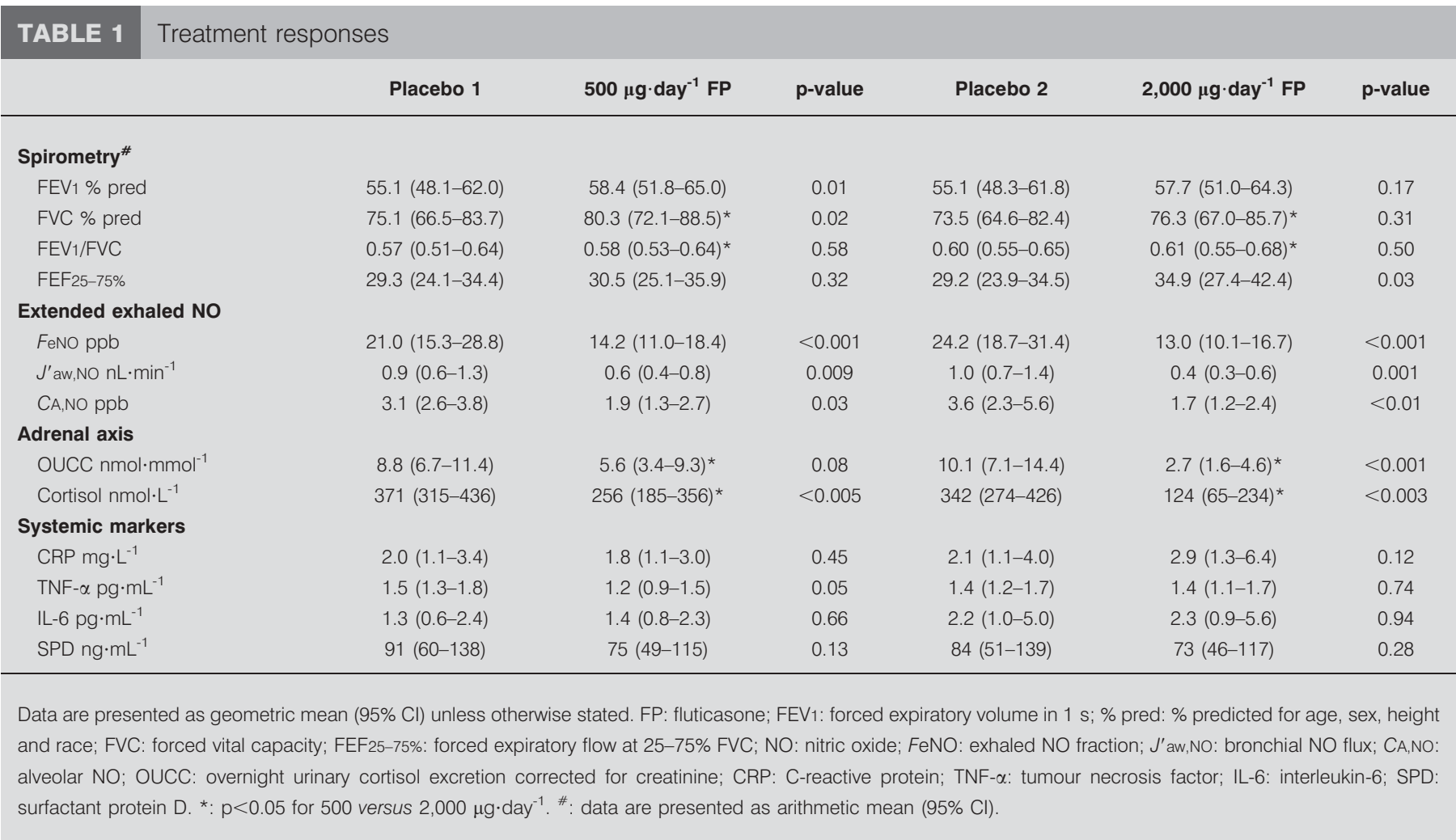

insight into whether this reflected suppression of airway inflammation or systemic adrenal suppression. We observed suppression of $\mathrm{FeNO}, J^{\prime}$ aw,NO and $\mathrm{CA}$,NO with both doses of FP, and there was no significant dose response. Since cortisol was suppressed even at $500 \mu \mathrm{g} \cdot \mathrm{day}^{-1} \mathrm{FP}$, we can not exclude a systemic effect to explain $\mathrm{CA}$,NO suppression, rather than fine particle dose deposition into the small airways.

The only outcome to show both suppression with $500 \mu \mathrm{g} \cdot \mathrm{day}^{-1}$ $\mathrm{FP}$, and a dose response between moderate and high doses was morning cortisol. Similarly, OUCC showed a dose response and trend to suppression on $500 \mu \mathrm{g} \cdot$ day $^{-1}$ FP $\quad(p=0.08)$. Although this is the most direct marker of steroid activity, it re-enforces the potential harm of long-term ICS exposure relative to inflammation. This study highlights that, despite long term benefits with ICS on exacerbations, COPD remains a relatively steroid-unresponsive condition, with a far greater dose-response relationship to adrenal suppression than systemic or airway inflammation.

There are limitations to our study design that should be addressed. The study was small with relatively short treatment periods, though power calculations and duration were based on published data available at the time of conception [8]. As treatment periods were short, we chose a dose and device to maximise airway deposition, such that a potent systemic antiinflammatory effect would be achieved. Whilst parallel group design is desirable, COPD is a highly heterogenous condition with variation in symptoms relative to inflammation and airflow obstruction. To reduce the influence of such heterogeneity, we opted for a crossover design. Despite these limitations, we hope our data will stimulate more widespread consideration of what is known about the optimal dose of ICS in COPD.

It is current practice to prescribe high-dose ICS in moderate-tosevere COPD; however, the appropriate dose for long-term therapy has not been established. The dose-response relationship of ICS is marked on adrenal suppression compared to biomarkers of systemic and airway inflammation. Further long-term dose-ranging studies are required to establish the risk/benefit ratio of ICS in COPD.

\section{P.A. Williamson, D. Menzies, K.L. Clearie, S. Vaidyanathan and B.J. Lipworth}

Asthma and Allergy Research Group, University of Dundee, Ninewells Hospital and Medical School, Dundee, UK.

Correspondence: B.J. Lipworth, Asthma and Allergy Research Group, University of Dundee, Ninewells Hospital and Medical School, Dundee, DD1 9SY, UK. E-mail: brianlipworth@ googlemail.com

Statement of Interest: A statement of interest for D. Menzies can be found at www.erj.ersjournals.com/site/misc/statements. xhtml

\section{REFERENCES}

1 Calverley PM, Anderson JA, Celli B, et al. Salmeterol and fluticasone propionate and survival in chronic obstructive pulmonary disease. N Engl J Med 2007; 356: 775-789. 
2 Sin DD, Wu L, Anderson JA, et al. Inhaled corticosteroids and mortality in chronic obstructive pulmonary disease. Thorax 2005; 60: 992-997.

3 Weatherall M, Clay J, James K, et al. Dose-response relationship of inhaled corticosteroids and cataracts: a systematic review and meta-analysis. Respirology 2009; 14: 983-990.

4 Weatherall M, James K, Clay J, et al. Dose-response relationship for risk of non-vertebral fracture with inhaled corticosteroids. Clin Exp Allergy 2008; 38: 1451-1458.

5 Hurst JR, Donaldson GC, Quint JK, et al. Temporal clustering of exacerbations in chronic obstructive pulmonary disease. Am J Respir Crit Care Med 2009; 179: 369-374.

6 Pietropaoli AP, Perillo IB, Perkins PT, et al. Smokers have reduced nitric oxide production by conducting airways but normal levels in the alveoli. Inhal Toxicol 2007; 19: 533-541.
7 Tsoukias NM, George SC. A two-compartment model of pulmonary nitric oxide exchange dynamics. J Appl Physiol 1998; 85: 653-666.

8 Sin DD, Lacy P, York E, et al. Effects of fluticasone on systemic markers of inflammation in chronic obstructive pulmonary disease. Am J Respir Crit Care Med 2004; 170: 760-765.

9 Masoli M, Weatherall M, Holt S, et al. Inhaled fluticasone propionate and adrenal effects in adult asthma: systematic review and meta-analysis. Eur Respir J 2006; 28: 960-967.

10 Sin DD, Man SF, Marciniuk DD, et al. The effects of fluticasone with or without salmeterol on systemic biomarkers of inflammation in chronic obstructive pulmonary disease. Am J Respir Crit Care Med 2008; 177: 1207-1214.

\section{Dose-dependent effects of mandibular protrusion on genioglossus activity in sleep apnoea}

\section{To the Editors:}

In individuals with a compromised upper airway, the activity of the genioglossus (GG) muscle is augmented to maintain upper airway patency during wakefulness [1]. However, sleep greatly suppresses GG muscle activity, which may lead to partial or complete upper airway collapse in patients with obstructive sleep apnoea (OSA). Mandibular-advancing oral appliances are currently accepted as one promising approach to the treatment of mild-to-moderate OSA [2, 3]. Although enlargement of the upper airway at multiple levels may be important for producing a clinical effect, the contributions of oral appliances to functional aspects, in terms of GG muscle activity, have hardly been documented.

If mandibular advancement increased the electromyographic activity of the GG muscle (GGEMG), this effect should help to maintain the patency of the airway, since the activation of GGEMG could stiffen the compliant airway wall [4]. In contrast, if we consider that mandibular advancement increases the cross-sectional area and decreases the propensity for upper airway occlusion, it would be reasonable to hypothesise that a larger, less collapsible upper airway could improve airflow dynamics, which would require less GGEMG [5]. Accordingly, we investigated the effects of mandibular advancement by an experimental oral appliance on GGEMG and the severity of OSA during sleep.

The study protocol was approved by the Clinical Research Ethics Board at the University of British Columbia (UBC), Vancouver, Canada. A 54-yr-old male patient with severe OSA (body mass index $31.2 \mathrm{~kg} \cdot \mathrm{m}^{-2}$ and apnoea/hypopnoea index (AHI) 30.3 events $\cdot \mathrm{hr}^{-1}$ ) who showed a resolution of symptoms, including daytime sleepiness, snoring and/or apnoea, after complete titration of the oral appliance (Klearway ${ }^{\mathrm{TM}}$; Great Lakes Orthodontics, Tonawanda, NY, USA) was recruited into the study. The patient had used the oral appliance for a period of 4 months prior to the study. The details of the study aims and methods were explained, and written informed consent was obtained.

A custom-made experimental oral appliance was fabricated at the most retruded mandibular position with a vertical opening of the mandible of $2 \mathrm{~mm}$ between the upper and lower incisors. The experimental oral appliance incorporated a pair of custom-made silver/silver chloride ball-type surface electrodes located lingually to record GGEMG [6]. Despite the known significant limitations of surface electrodes compared with needle electrodes, in that the former might pick up geniohyoid and/or mylohyoid muscle activities to some extent [7], we chose to use the surface GG recording technique because it is noninvasive and we were also considering possible future clinical use. A remote-controlled mandibular positioner (RCMP; SagaTech Electronics Inc., Calgary, Canada) was connected to the experimental appliance to reproduce passive forward mandibular movement (see online supplementary material) $[8,9]$. In the first waking session, the patient was asked to insert the experimental oral appliance, to confirm whether the electrodes recorded the GGEMG signal and whether passive mandibular movement with the RCMP was comfortable. Recording was performed from the most retruded mandibular position to the maximum comfortable limit of mandibular advancement with a 1-mm increment forward in the supine body position. Movement of the ribcage was recorded simultaneously. The raw GGEMG signal was monitored with an oscilloscope, in order to confirm a clear and stable inspiratory phasic GGEMG [6]. Thereafter, the patient was scheduled to undergo an overnight polysomnographic sleep study at the sleep laboratory of the UBC Hospital, Vancouver. The same experimental oral appliance with the same recording procedure as in the waking session was used 